\title{
A trace partitioned Gray code for $q$-ary generalized Fibonacci strings
}

\author{
A. Bernini, S. Bilotta, R. Pinzani \\ Dipartimento di Matematica e Informatica "Ulisse Dini" \\ Università degli Studi di Firenze, Viale G.B. Morgagni 65, 50134 Firenze, Italy \\ e-mail: \{antonio.bernini\}\{stefano.bilotta\}\{renzo.pinzani@unifi\}@unifi.it \\ V. Vajnovszki \\ LE2I, Université de Bourgogne, BP 47 870, 21078 Dijon Cedex, France \\ e-mail: vvajnov@u-bourgogne.fr \\ July 13, 2018
}

\begin{abstract}
We provide a trace partitioned Gray code for the set of $q$-ary strings avoiding a pattern constituted by $k$ consecutive equal symbols. The definition of this Gray code is based on two different constructions, according to the parity of $q$. This result generalizes, and is based on, a Gray code for binary strings avoiding $k$ consecutive 0's.
\end{abstract}

Keyword: Gray codes, pattern avoiding strings, generalized Fibonacci numbers

\section{Introduction}

The famous $k$-generalized Fibonacci sequence $\left\{f_{n}^{(k)}\right\}_{n \geq 0}$ is defined by

$$
f_{n}^{(k)}=f_{n-1}^{(k)}+f_{n-2}^{(k)}+\cdots+f_{n-k}^{(k)}, \text { for } n \geq k,
$$

with initial conditions [4]: $f_{i}^{(k)}=0$ for $0 \leq i \leq k-2$, and $f_{k-1}^{(k)}=1$. This sequence is related to the enumeration of binary strings avoiding $k$ consecutive 1 's [4], called $k$-generalized Fibonacci strings.

If an alphabet of cardinality $q \geq 2$ is used, then the enumeration of the strings of length $n$ and avoiding a pattern constituted by $k$ consecutive occurrences of a same symbol is given by

$$
f_{n, q}^{(k)}=(q-1)\left(f_{n-1, q}^{(k)}+f_{n-2, q}^{(k)}+\ldots+f_{n-k, q}^{(k)}\right), \text { for } n \geq k,
$$

with $f_{i, q}^{(k)}=q^{i}$, for $0 \leq i \leq k-1$; and in particular $f_{n+k}^{(k)}=f_{n, 2}^{(k)}$. This sequence is a particular case of the weighted k-generalized Fibonacci sequence (studied and used in [5, 7]) where all the weights are equal to $q-1$. Similarly to the binary case, by a $q$-ary $k$-generalized Fibonacci string we mean a string over a $q \geq 2$ letter alphabet $A$ and avoiding $k$ consecutive occurrences of a given symbol of $A$. For example, for $k=3$ and $A=\{0,1,2,3\}, 111$ or 222 is avoided, but not both. 
Once a class of objects is defined, often it could be of interest to list or generate them according to a particular criterion. A special way to do this is to generate the objects so that any two consecutive ones differ as little as possible, i.e., in a Gray code manner [2]. Such a particular code has already been proposed for (binary) $k$-generalized Fibonacci strings [9].

In the present work we provide a Gray code for the $q$-ary $k$-generalized Fibonacci strings, $q \geq 2$, which extends the approach in [9]. Our method gives a trace partitioned code where strings with the same trace are contiguous (for more details see Section 21). Analogously to the kind of the forbidden pattern considered in [9] (which is a sequence of $k$ consecutive 1's), here we consider the avoidance of $k$ consecutive occurrences of a give symbol, not necessarily 1 , of a $q$-ary alphabet.

If $L$ is a set of strings over an alphabet $A$, then by $\mathcal{L}$ we denote the ordered list where the strings of $L$ are listed following a certain criterion. If the Hamming distance [3] between two successive elements of $\mathcal{L}$ is bounded by a constant, than $\mathcal{L}$ is called Gray code list. The notations we are going to use are defined below:

- if $\alpha$ and $\beta$ are two same length strings, then $d_{H}(\alpha, \beta)$ denotes their Hamming distance;

- $\overline{\mathcal{L}}$ denotes the list obtained by covering $\mathcal{L}$ in reverse order;

- first $(\mathcal{L})$ and last $(\mathcal{L})$ are the first and the last element of $\mathcal{L}$, respectively, and clearly first $(\mathcal{L})=\operatorname{last}(\overline{\mathcal{L}})$, and last $(\mathcal{L})=\operatorname{first}(\overline{\mathcal{L}}) ;$

- if $u$ is a string, then $u \cdot \mathcal{L}$ is the list obtained by prepending $u$ to each string in $\mathcal{L}$;

- if $\mathcal{L}$ and $\mathcal{L}^{\prime}$ are two lists, then $\mathcal{L} \circ \mathcal{L}^{\prime}$ is the concatenation of the two lists, obtained by appending the elements of $\mathcal{L}^{\prime}$ after the elements of $\mathcal{L}$.

\section{Gray codes}

The well known Binary Reflected Gray Code (BRGC) [2] can be generalized to an alphabet of cardinality greater than 2 [1, 10]. If $A=\left\{a_{0}, a_{1}, \ldots, a_{q-1}\right\}$, then the list $\mathcal{G}_{n}^{q}$ of the length $n$ strings over $A$ is given by:

$$
\mathcal{G}_{n}^{q}=\left\{\begin{array}{cl}
\lambda & \text { if } \quad n=0, \\
a_{0} \cdot \mathcal{G}_{n-1}^{q} \circ a_{1} \cdot \overline{\mathcal{G}_{n-1}^{q}} \circ \cdots \circ a_{q-1} \cdot \mathcal{G}_{n-1}^{\prime} q & \text { if } \quad n>0,
\end{array}\right.
$$

where $\mathcal{G}_{n-1}^{q}$ is $\overline{\mathcal{G}_{n-1}^{q}}$ if $q$ is even or $\mathcal{G}_{n-1}^{q}$ if $q$ is odd, and $\lambda$ is the empty string. It is proved that $\mathcal{G}_{n}^{q}$ is a Gray code list with Hamming distance 1 and the reader can easily prove the following proposition.

Proposition 1. If $q$ is odd, then last $\left(\mathcal{G}_{n}^{q}\right)=a_{q-1}^{n}$ and first $\left(\mathcal{G}_{n}^{q}\right)=a_{0}^{n}$. In particular,

$$
\operatorname{last}\left(\mathcal{G}_{n}^{q}\right)=\operatorname{last}\left(\mathcal{G}_{n-1}^{q}\right) \cdot a_{q-1}=a_{q-1} \cdot \operatorname{last}\left(\mathcal{G}_{n-1}^{q}\right),
$$

and

$$
\operatorname{first}\left(\mathcal{G}_{n}^{q}\right)=\operatorname{first}\left(\mathcal{G}_{n-1}^{q}\right) \cdot a_{0}=a_{0} \cdot \operatorname{first}\left(\mathcal{G}_{n-1}^{q}\right)
$$


If $q$ is even, then last $\left(\mathcal{G}_{n}^{q}\right)=a_{q-1} a_{0}^{n-1}$ and first $\left(\mathcal{G}_{n}^{q}\right)=a_{0}^{n}$. In particular, for $n>1$,

$$
\operatorname{last}\left(\mathcal{G}_{n}^{q}\right)=a_{q-1} a_{0}^{n-1}=\operatorname{last}\left(\mathcal{G}_{n-1}^{q}\right) \cdot a_{0},
$$

and

$$
\operatorname{first}\left(\mathcal{G}_{n}^{q}\right)=a_{0}^{n}=\operatorname{first}\left(\mathcal{G}_{n-1}^{q}\right) \cdot a_{0}=a_{0} \cdot \operatorname{first}\left(\mathcal{G}_{n-1}^{q}\right) .
$$

We will give a Gray code for the set of $q$-ary $k$-generalized Fibonacci strings of length $n$, where the Hamming distance between two consecutive strings is 1. The definition of our Gray code depends on the parity of $q$, and we will refer simply to Gray code for a list where two successive elements have Hamming distance equal to 1 , and without any loss of generality we will consider the alphabet $A=\{0,1, \ldots, q-1\}$. For our topics we are going to consider the avoidance of the pattern $0^{k}$, for a given $k \geq 2$, but all of our constructions can be easily translated to the pattern $i^{k}$, for any $i \in A$.

Our approach starts from the definition of the Gray code for binary $k$-generalized Fibonacci strings given in [9], then using a bit replacing technique we extend the binary alphabet to $A$, leading to a trace partitioned Gray code (where strings with the same trace are consecutive). The trace of a $q$-ary string is a binary string obtained by replacing each symbol different from 0 by 1 .

Before going along in our discussion, we define a tool for manipulating the symbols of a string. For $q \geq 3$, we denote by $\mathcal{G}_{t}^{q-1} \oplus 1$ the list obtained from $\mathcal{G}_{t}^{q-1}$ by adding 1 to each symbol in each string in $\mathcal{G}_{t}^{q-1}$. Actually, $\mathcal{G}_{t}^{q-1} \oplus 1$ is the Gray code defined in relation (11) for $\left\{a_{0}, a_{1}, \ldots, a_{q-1}\right\}=\{1,2, \ldots, q\}$. For example, $\mathcal{G}_{3}^{2} \oplus 1=(111,112,122,121,221,222,212,211)$.

If $\beta$ is a binary string of length $n$ such that $|\beta|_{1}=t$ (the number of 1 's in $\beta$ ), we define the expansion of $\beta$, denoted by $\varepsilon(\beta)$, as the list of $(q-1)^{t}$ strings, where the $i$-th string is obtained by replacing the $t$ 1's of $\beta$ by the $t$ symbols (read from left to right) of the $i$-th string in $\mathcal{G}_{t}^{q-1} \oplus 1$. For example, if $q=3$ and $\beta=01011$ (the trace), then with $\mathcal{G}_{3}^{2} \oplus 1$ given above, we have $\varepsilon(\beta)=(01011,01012,01022,01021,02021,02022,02012,02011)$. Notice that in particular first $(\varepsilon(\beta))=\beta$ and all the elements of $\varepsilon(\beta)$ have the same trace.

We observe that $\varepsilon(\beta)$ is the list obtained from $\mathcal{G}_{t}^{q-1}$ by adding 1 to each symbol of each string in $\mathcal{G}_{t}^{q-1}$, then inserting some 0 's, each time in the same positions. Since $\mathcal{G}_{t}^{q-1}$ is a Gray code and the insertions of the 0 's does not change the Hamming distance between two successive element of $\varepsilon(\beta)$ (which is 1 ), we have the following:

Proposition 2. For any $q \geq 3$ and binary string $\beta$, the list $\varepsilon(\beta)$ is a Gray code.

The Gray code we are going to consider as the starting point of our argument is the one defined in [9], where the author deals with binary strings avoiding $k$ consecutive 1's. Since we are interested in the avoidance of $k$ consecutive 0's, we recall, for the sake of clearness, the definition in [9] adapted according to our needs which cause some slight differences with respect to the original definition in $[9$.

Let $\mathcal{F}_{n}^{(k)}$ be the list defined as:

$$
\mathcal{F}_{n}^{(k)}=\left\{\begin{array}{cr}
\mathcal{C}_{n} & \text { if } 0 \leq n<k, \\
1 \cdot \overline{\mathcal{F}_{n-1}^{(k)}} \circ 01 \cdot \overline{\mathcal{F}_{n-2}^{(k)}} \circ 001 \cdot \overline{\mathcal{F}_{n-3}^{(k)}} \circ \ldots \circ 0^{k-1} 1 \cdot \overline{\mathcal{F}_{n-k}^{(k)}} & \text { if } n \geq k,
\end{array}\right.
$$


where

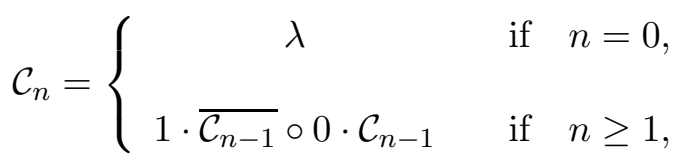

with $\lambda$ the empty string.

It is proved [9] that $\mathcal{F}_{n}^{(k)}$ is a Gray code for the set of binary length $n$ strings avoiding $k$ consecutive 0's, and thus the number of strings in $\mathcal{F}_{n}^{(k)}$ is $f_{n+k}^{(k)}$, the $(n+k)$-th value of the $k$-generalized Fibonacci sequence.

Now let $F_{n, q}^{(k)}$ be the set of length $n$ strings over $A=\{0,1, \ldots, q-1\}, q>2$, avoiding $k$ consecutive 0's, so $\left|F_{n, q}^{(k)}\right|=f_{n, q}^{(k)}$ (see Introduction). The aim is the construction of a Gray code for $F_{n, q}^{(k)}$. Our definition of such a Gray code is based on the expansion $\varepsilon\left(\alpha_{i}\right)$ (or its reverse $\left.\overline{\varepsilon\left(\alpha_{i}\right)}\right)$ of each element of $\mathcal{F}_{n}^{(k)}=\left(\alpha_{1}, \alpha_{2}, \ldots, \alpha_{f_{n+k}^{(k)}}\right)$, and then concatenating them opportunely, according to the parity of $q$.

Let us illustrate the construction of the list for the set $F_{n, q}^{(k)}$, of the form

$$
\varepsilon\left(\alpha_{1}\right) \circ \overline{\varepsilon\left(\alpha_{2}\right)} \circ \varepsilon\left(\alpha_{3}\right) \circ \overline{\varepsilon\left(\alpha_{4}\right)} \ldots
$$

where $\alpha_{i}$ covers the list $\mathcal{F}_{n}^{(k)}$. As we will see below, this construction yields a Gray code when $q$ is even, but not necessarily when $q$ is odd.

When $k=q=n=3$, the list $\mathcal{F}_{n}^{(k)}$ is $\mathcal{F}_{3}^{(3)}=(100,101,111,110,010,011,001) ; \mathcal{G}_{1}^{2} \oplus 1=(1,2)$, $\mathcal{G}_{2}^{2} \oplus 1=(11,12,22,21)$, and $\mathcal{G}_{3}^{2} \oplus 1$ is given in the example preceding Proposition 2 2 The expansions of the elements of $\mathcal{F}_{3}^{(3)}$ are:

$$
\begin{aligned}
& \varepsilon\left(\alpha_{1}\right)=\varepsilon(100)=(100,200) \\
& \varepsilon\left(\alpha_{2}\right)=\varepsilon(101)=(101,102,202,201) \\
& \varepsilon\left(\alpha_{3}\right)=\varepsilon(111)=(111,112,122,121,221,222,212,211) \\
& \varepsilon\left(\alpha_{4}\right)=\varepsilon(110)=(110,120,220,210) \\
& \varepsilon\left(\alpha_{5}\right)=\varepsilon(010)=(010,020) \\
& \varepsilon\left(\alpha_{6}\right)=\varepsilon(011)=(011,012,022,021) \\
& \varepsilon\left(\alpha_{7}\right)=\varepsilon(001)=(001,002),
\end{aligned}
$$

and the list of the form (3) for $F_{3,3}^{(3)}$ is

$$
\varepsilon\left(\alpha_{1}\right) \circ \overline{\varepsilon\left(\alpha_{2}\right)} \circ \varepsilon\left(\alpha_{3}\right) \circ \overline{\varepsilon\left(\alpha_{4}\right)} \circ \varepsilon\left(\alpha_{5}\right) \circ \overline{\varepsilon\left(\alpha_{6}\right)} \circ \varepsilon\left(\alpha_{7}\right)
$$

which is a Gray code, as it easily can be checked. However, this is not true in general. For example, if $n=4, k=q=3$, concatenating $\epsilon\left(\alpha_{i}\right)$ and $\overline{\varepsilon\left(\alpha_{i+1}\right)}$, alternatively as in (3) does not yield a Gray code. Indeed,

- $\alpha_{7}=1100$ and $\alpha_{8}=0100$, and

- $\varepsilon\left(\alpha_{7}\right)=(1100,1200,2200,2100)$ and $\varepsilon\left(\alpha_{8}\right)=(0100,0200)$.

And in the concatenation

$\varepsilon\left(\alpha_{1}\right) \circ \overline{\varepsilon\left(\alpha_{2}\right)} \circ \varepsilon\left(\alpha_{3}\right) \circ \overline{\varepsilon\left(\alpha_{4}\right)} \circ \varepsilon\left(\alpha_{5}\right) \circ \overline{\varepsilon\left(\alpha_{6}\right)} \circ \varepsilon\left(\alpha_{7}\right) \circ \overline{\varepsilon\left(\alpha_{8}\right)} \circ \varepsilon\left(\alpha_{9}\right) \circ \overline{\varepsilon\left(\alpha_{10}\right)} \circ \varepsilon\left(\alpha_{11}\right) \circ \overline{\varepsilon\left(\alpha_{12}\right)} \circ \varepsilon\left(\alpha_{13}\right)$,

in the transition from $\varepsilon\left(\alpha_{7}\right)$ to $\overline{\varepsilon\left(\alpha_{8}\right)}$ we found 2100 followed by 0200 which differ in more than one position. 


\subsection{The case of $q$ odd}

A way to overcome the previous difficulties is to consider the partition of $\mathcal{F}_{n}^{(k)}$ as in its definition (2). For $j=1,2, \ldots, k$, let $\alpha_{i}^{(j)}$ be the $i$-th element in the list $0^{j-1} 1 \cdot \overline{\mathcal{F}_{n-j}^{(k)}}$, and

$$
\Gamma_{j}=\varepsilon\left(\alpha_{1}^{(j)}\right) \circ \overline{\varepsilon\left(\alpha_{2}^{(j)}\right)} \circ \varepsilon\left(\alpha_{3}^{(j)}\right) \circ \overline{\varepsilon\left(\alpha_{4}^{(j)}\right)} \circ \ldots \circ \varepsilon^{\prime}\left(\alpha_{f_{n+k-j}^{(j)}}^{(j)}\right),
$$

where $\varepsilon^{\prime}\left(\alpha_{f_{n+k-j}^{(k)}}^{(j)}\right)=\varepsilon\left(\alpha_{f_{n+k-j}^{(k)}}^{(j)}\right)$ if $f_{n+k-j}^{(k)}$ is odd and $\varepsilon^{\prime}\left(\alpha_{f_{n+k-j}^{(k)}}^{(j)}\right)=\overline{\varepsilon\left(\alpha_{f_{n+k-j}^{(k)}}^{(j)}\right)}$ if $f_{n+k-j}^{(k)}$ is even. Let define $\mathcal{F}_{n, q}^{(k)}$ as

$$
\mathcal{F}_{n, q}^{(k)}=\Gamma_{1} \circ \Gamma_{2} \circ \ldots \circ \Gamma_{k},
$$

and clearly $\mathcal{F}_{n, q}^{(k)}$ is a list for the set $F_{n, q}^{(k)}$ and the next proposition proves that it is a Gray code.

Proposition 3. If $q$ is odd, then the list $\mathcal{F}_{n, q}^{(k)}=\Gamma_{1} \circ \Gamma_{2} \circ \ldots \circ \Gamma_{k}$ is a Gray code list with Hamming distance 1.

Proof. We have to prove the following:

1. $\Gamma_{j}$ is a Gray code list, for each $j=1,2, \ldots, k$, with Hamming distance 1 ;

2. $d_{H}\left(\operatorname{last}\left(\Gamma_{j}\right), \operatorname{first}\left(\Gamma_{j+1}\right)\right)=1$, for $j=1,2, \ldots, k-1$.

By Proposition 2 it follows that $\varepsilon\left(\alpha_{i}^{(j)}\right)$ is a Gray code, and for the point 1 we have to check that, if $i$ is odd, $d_{H}\left(\operatorname{last}\left(\varepsilon\left(\alpha_{i}^{(j)}\right)\right)\right.$, first $\left.\left(\overline{\varepsilon\left(\alpha_{i+1}^{(j)}\right)}\right)\right)=1$ and that, if $i$ is even, $d_{H}\left(\operatorname{last}\left(\overline{\varepsilon\left(\alpha_{i}^{(j)}\right)}\right)\right.$, first $\left.\left(\varepsilon\left(\alpha_{i+1}^{(j)}\right)\right)\right)=$ 1 .

- When $i$ is odd we observe that, for some $v$ and $w$,

$$
\begin{aligned}
\alpha_{i}^{(j)} & =0^{j-1} 1 v, \text { and } \\
\alpha_{i+1}^{(j)} & =0^{j-1} 1 w
\end{aligned}
$$

where $v$ and $w$ differ in a single position since $\alpha_{i}^{(j)}$ and $\alpha_{i+1}^{(j)}$ are two consecutive binary strings in $\mathcal{F}_{n}^{(k)}$, which is a Gray code list.

Let $t=|v|_{1}$, and since $q-1$ is even, by Proposition 1 it follows that last $\left(\mathcal{G}_{t+1}^{q-1}+1\right)=(q-1) 1^{t}$ which occurs in the last element of the expansion of $\alpha_{i}^{(j)}$. Therefore, last $\left(\varepsilon\left(\alpha_{i}^{(j)}\right)\right)=$ $0^{j-1}(q-1) v$.

Now, first $\left.\overline{\left(\varepsilon\left(\alpha_{i+1}^{(j)}\right)\right.}\right)$ is equal to last $\left(\varepsilon\left(\alpha_{i+1}^{(j)}\right)\right)$, which as previously, is equal in turn to $0^{j-1}(q-$ 1) $w$.

Since $v$ and $w$ differ in a single position, so do last $\left(\varepsilon\left(\alpha_{i}^{(j)}\right)\right)$ and first $\left.\overline{\varepsilon\left(\alpha_{i+1}^{(j)}\right)}\right)$.

- If $i$ is even, and since last $\left.\overline{\varepsilon\left(\alpha_{i}^{(j)}\right)}\right)=$ first $\left(\varepsilon\left(\alpha_{i}^{(j)}\right)\right)$, by the definition of expansion it follows that

$$
\begin{aligned}
& \operatorname{first}\left(\varepsilon\left(\alpha_{i}^{(j)}\right)\right)=\alpha_{i}^{(j)}, \text { and } \\
& \operatorname{first}\left(\varepsilon\left(\alpha_{i+1}^{(j)}\right)\right)=\alpha_{i+1}^{(j)} .
\end{aligned}
$$


Since $\alpha_{i}^{(j)}$ and $\alpha_{i+1}^{(j)}$ are two consecutive strings, their Hamming distance is 1 .

For the second point, we have

$$
\begin{aligned}
\operatorname{first}\left(\Gamma_{j+1}\right) & =\operatorname{first}\left(\varepsilon\left(\alpha_{1}^{(j+1)}\right)\right), \text { and } \\
\operatorname{last}\left(\Gamma_{j}\right) & =\operatorname{last}\left(\varepsilon^{\prime}\left(\alpha_{f_{n+k-j}^{(k)}}^{(j)}\right)\right)
\end{aligned}
$$

where, for some $w^{\prime}$ and $w^{\prime \prime}$,

$$
\begin{aligned}
\alpha_{1}^{(j+1)} & =0^{j} 1 w^{\prime}, \text { and } \\
\alpha_{f_{n+k-j}^{(k)}}^{(j)} & =0^{j-1} 1 w^{\prime \prime} .
\end{aligned}
$$

Since $\alpha_{f_{n+k-j}^{(k)}}^{(j)}$ and $\alpha_{1}^{(j+1)}$ are two consecutive strings in $\mathcal{F}_{n}^{(k)}$, their Hamming distance is 1 , and thus $w^{\prime \prime}=1 w^{\prime}$. Two cases can occur:

- if $f_{n+k-j}^{(k)}$ is even, then last $\left(\Gamma_{j}\right)=\operatorname{last}\left(\varepsilon^{\prime}\left(\alpha_{f_{n+k-j}^{(k)}(j)}^{(k)}\right)\right)=\operatorname{last}\left(\overline{\left(\alpha_{f_{n+k-j}^{(k)}}^{(j)}\right)}\right)=\operatorname{first}\left(\varepsilon\left(\alpha_{f_{n+k-j}^{(k)}}^{(j)}\right)\right)=$ $\alpha_{f_{n+k-j}^{(k)}}^{(j)}$. Moreover, first $\left(\Gamma_{j+1}\right)=\operatorname{first}\left(\varepsilon\left(\alpha_{1}^{(j+1)}\right)\right)=\alpha_{1}^{(j+1)}$.

- if $f_{n+k-j}^{(k)}$ is odd, then $\operatorname{last}\left(\Gamma_{j}\right)=\operatorname{last}\left(\varepsilon\left(\alpha_{f_{n+k-j}^{(k)}}^{(j)}\right)\right)=0^{j-1}(q-1) w^{\prime \prime}$ and $\operatorname{first}\left(\Gamma_{j+1}\right)=$ first $\left(\varepsilon\left(\alpha_{1}^{(j+1)}\right)\right)=\alpha_{1}^{(j+1)}=0^{j} 1 w^{\prime}=0^{j} w^{\prime \prime}$.

In any case, $d_{H}\left(\operatorname{last}\left(\Gamma_{j}\right)\right.$, first $\left.\left(\Gamma_{j+1}\right)\right)=1$.

Therefore the proof is concluded and $\mathcal{F}_{n, q}^{(k)}$ is a Gray code list with Hamming distance 1.

It is easy to see that, generally, when $q$ is even, the construction given in the previous proposition does not yield a Gray code.

For the sake of clearness, we illustrate the previous construction for the Gray code when $n=4, k=3$ and $A=\{0,1,2\}$. We have:

$$
\begin{aligned}
\mathcal{F}_{4}^{(3)}= & (\underbrace{1001,1011,1010,1110,1111,1101,1100}_{\alpha_{i}^{(1)} \in 1 \cdot \overline{\mathcal{F}_{3}^{(3)}}}, \underbrace{0100,0101,0111,0110}_{\alpha_{i}^{(2)} \in 01 \cdot \overline{\mathcal{F}_{2}^{(3)}}}, \underbrace{0010,0011}_{\alpha_{i}^{(3)} \in 001 \cdot \overline{\mathcal{F}_{1}^{(3)}}}) \\
\mathcal{G}_{0}^{2} \oplus 1= & \lambda ; \\
\mathcal{G}_{1}^{2} \oplus 1= & (1,2) ; \\
\mathcal{G}_{2}^{2} \oplus 1= & (11,12,22,21) ; \\
\mathcal{G}_{3}^{2} \oplus 1= & (111,112,122,121,221,222,212,211) ; \\
\mathcal{G}_{4}^{2} \oplus 1= & (1111,1112,1122,1121,1221,1222,1212,1211,2211,2212,2222,2221 \\
& 2121,2122,2112,2111) ;
\end{aligned}
$$




$$
\begin{aligned}
& \Gamma_{1}=(\underbrace{1001,1002,2002,2001}_{\varepsilon\left(\alpha_{1}^{(1)}\right)}, \underbrace{2011, \ldots, 1011}_{\overline{\varepsilon\left(\alpha_{2}^{(1)}\right)}}, \underbrace{1010, \ldots, 2010}_{\varepsilon\left(\alpha_{3}^{(1)}\right)}, \underbrace{2110}_{\frac{\varepsilon\left(\alpha_{4}^{(1)}\right)}{2110, \ldots, 1110}} \\
& \underbrace{1111, \ldots, 2111}_{\varepsilon\left(\alpha_{5}^{(1)}\right)} \underbrace{2100, \ldots, 2100}_{\frac{\left.\alpha_{6}^{(1)}\right)}{2101, \ldots, 1101}}) \\
& \Gamma_{2}=(\underbrace{0100,0200}_{\varepsilon\left(\alpha_{1}^{(2)}\right)}, \underbrace{0201, \ldots, 0101}_{\overline{\varepsilon\left(\alpha_{2}^{(2)}\right)}} \underbrace{0111, \ldots, 0211}_{\varepsilon\left(\alpha_{3}^{(2)}\right)} \underbrace{021, \ldots}_{\frac{\varepsilon\left(\alpha_{4}^{(2)}\right)}{0210, \ldots, 0110}} \\
& \Gamma_{3}=(\underbrace{0010,0020}_{\varepsilon\left(\alpha_{1}^{(3)}\right)}, \underbrace{0021, \ldots, 0011}_{\overline{\varepsilon\left(\alpha_{2}^{(3)}\right)}}) .
\end{aligned}
$$

The reader can easily check that $\mathcal{F}_{4,3}^{(3)}=\Gamma_{1} \circ \Gamma_{2} \circ \Gamma_{3}$ is a Gray code with Hamming distance 1.

\subsection{The case of $q$ even}

In this case the construction of a Gray code list for $F_{n, q}^{(k)}$ is straightforward, and based on the discussion at the beginning of this section: just consider the expansions of the binary strings $\alpha_{i}$ in $\mathcal{F}_{n}^{(k)}$, for $i=1,2, \ldots, f_{n+k}^{(k)}$, and concatenate them, taking $\varepsilon\left(\alpha_{i}\right)$ and $\overline{\varepsilon\left(\alpha_{i+1}\right)}$ alternatively, as in expression (3). The next proposition shows that the obtained list is a Gray code.

Proposition 4. If $q$ is even, then the list

$$
\mathcal{F}_{n, q}^{(k)}=\varepsilon\left(\alpha_{1}\right) \circ \overline{\varepsilon\left(\alpha_{2}\right)} \circ \ldots \circ \varepsilon^{\prime}\left(\alpha_{f_{n+k}^{(k)}}\right),
$$

where $\varepsilon^{\prime}\left(\alpha_{f_{n+k}^{(k)}}\right)=\varepsilon\left(\alpha_{f_{n+k}^{(k)}}\right)$ if $f_{n+k}^{(k)}$ is odd and $\varepsilon^{\prime}\left(\alpha_{f_{n+k}^{(k)}}\right)=\overline{\varepsilon\left(\alpha_{f_{n+k}^{(k)}}\right)}$ if $f_{n+k}^{(k)}$ is even, is a Gray code list with Hamming distance 1.

Proof. By Proposition 2, each $\varepsilon\left(\alpha_{i}\right)$ is a Gray code list, and, we have to check that, if $i$ is odd, $d_{H}\left(\operatorname{last}\left(\varepsilon\left(\alpha_{i}\right)\right)\right.$, first $\left.\left(\overline{\varepsilon\left(\alpha_{i+1}\right.}\right)\right)=1$ and that, if $i$ is even, $d_{H}\left(\operatorname{last}\left(\overline{\varepsilon\left(\alpha_{i}\right)}\right), \operatorname{first}\left(\varepsilon\left(\alpha_{i+1}\right)\right)\right)=1$.

- In the first case ( $i$ is odd), by Proposition 1 and the definition of expansion, it follows that in last $\left(\varepsilon\left(\alpha_{i}\right)\right)$ and in last $\left(\varepsilon\left(\alpha_{i+1}\right)\right)$ the symbols different from 0 are equal to $q-1$. For example, if $\alpha_{i}=10110$, then last $\left(\varepsilon\left(\alpha_{i}\right)\right)=(q-1) 0(q-1)(q-1) 0$. Moreover, since $\alpha_{i}$ and $\alpha_{i+1}$ are two consecutive strings of $\mathcal{F}_{n}^{(k)}, d_{H}\left(\alpha_{i}, \alpha_{i+1}\right)=1$, and so $d_{H}\left(\operatorname{last}\left(\varepsilon\left(\alpha_{i}\right)\right)\right.$, last $\left.\left(\varepsilon\left(\alpha_{i+1}\right)\right)\right)=1$.

- If $i$ is even, we observe that

$$
\begin{aligned}
\operatorname{last}\left(\overline{\varepsilon\left(\alpha_{i}\right)}\right) & =\operatorname{first}\left(\varepsilon\left(\alpha_{i}\right)\right)=\alpha_{i}, \text { and } \\
\operatorname{first}\left(\varepsilon\left(\alpha_{i+1}\right)\right) & =\alpha_{i+1} .
\end{aligned}
$$

Since $d_{H}\left(\alpha_{i}, \alpha_{i+1}\right)=1$, then $d_{H}\left(\operatorname{last}\left(\overline{\varepsilon\left(\alpha_{i}\right)}\right)\right.$, first $\left.\left(\varepsilon\left(\alpha_{i+1}\right)\right)\right)=1$. 


\section{Conclusions and further developments}

In this paper we propose a trace partitioned Gray code for the $q$-ary $k$-generalized Fibonacci strings of length $n$, where the Hamming distance between two successive strings is 1 . Our constructions are based on the expansion of an existing Gray code when $q=2$. A consequence of the expansion technique is that our Gray code has the following property: if we replace each non-zero symbol in each string by 1 , and 'collapse' the obtained list by keeping one copy of each binary strings, then the existing Gray code for $q=2$ is obtained.

The investigation on the existence of Gray codes for strings on a $q$-ary alphabet avoiding a general consecutive pattern has already been studied: in [8] the author gives such a Gray code only when $q$ even, while the case of $q$ odd is left open. Our Gray code deals with the avoidance of a particular pattern but works for any $q$, and an interesting development could be a deeper investigation to check if this constructions can be applied to a general consecutive pattern in order to solve the open question in [8]. Also, it would be of interest to implement our Gray code definition into an efficient generating algorithm for the set of underlying strings.

\section{References}

[1] Er, M. C.: On generating the $N$-ary reflected Gray code. IEEE Trans. Comput. 33 (1984) 739-741.

[2] Gray, F.: Pulse Code Communication U.S. Patent 2632058 (March 17, 1953).

[3] Hamming, R. W.: Error detecting and error correcting codes. Bell System Tech. J. 29 (1950) $147-160$.

[4] Knuth, D. E.: The Art of Computer Programming. Vol. 3, "Sorting and Searching", Addison-Wesley, Reading, MA, 1973.

[5] Levesque, C.: On m-th order linear recurrences. Fibonacci Quart. 23 (1985) 290-293.

[6] Ruskey, F.: Simple combinatorial Gray codes constructed by reversing sublists, 4th ISAAC, Lecture Notes in Computer Science, 762 (1993) 201-208.

[7] Shork, M.: The $r$-generalized Fibonacci numbers and Polynomial coefficients. Int. J. Contemp. Math. Sciences. 3 (2008) 1157-1163.

[8] Squire, M. B.: Gray codes for A-free strings. Electron. J. Combin. 3 (1996) \#R17.

[9] Vajnovszki, V.: A loopless generation of bitstrings without $p$ consecutive ones. Discrete Math. Theor. Comput. Sci. Springer 2001, 227-240.

[10] Williamson, S. G.: Combinatorics for computer science. Computer Science Press, Rockville, Maryland, 1985. 